\title{
Decidability for Justification Logics Revisited
}

\author{
Samuel Bucheli ${ }^{\star}$, Roman Kuznets ${ }^{\star \star}$, and Thomas Studer \\ Institut für Informatik und angewandte Mathematik \\ Universität Bern \\ \{bucheli, kuznets, tstuder\}@iam.unibe.ch \\ http://www.iam.unibe.ch/ltg
}

\begin{abstract}
Justification logics are propositional modal-like logics that instead of statements $A$ is known include statements of the form $A$ is known for reason $t$ where the term $t$ can represent an informal justification for $A$ or a formal proof of $A$. In our present work, we introduce model-theoretic tools, namely: filtrations and a certain form of generated submodels, in the context of justification logic in order to obtain decidability results. Apart from reproving already known results in a uniform way, we also prove new results. In particular, we use our submodel construction to establish decidability for a justification logic with common knowledge for which so far no decidability proof was available.
\end{abstract}

Keywords: Justification logic, decidability, filtration

\section{Introduction}

Justification logics are epistemic logics that explicitly include justifications for the agents' knowledge [3,4]. The first logic of this kind, the Logic of Proofs LP, was developed by Artemov to provide the modal logic S4 with provability semantics $[1,2]$. The language of justification logics has also been used to create a new approach to the logical omniscience problem [5], to study self-referential proofs [14], and to explore the evidential dynamics of public announcements [8, $10]$.

Instead of statements $A$ is known, denoted $\square A$, justification logics reason about justifications for knowledge by using the construct $t$ : $A$ to formalize statements $t$ is a justification for $A$, where, dependent on the application, the evidence term $t$ can be viewed as an informal justification or a formal mathematical proof. For an example see Fig. 1 where the axioms of the justification logic LP are listed alongside the axioms of S4 to point out the correspondence of the operations on evidence terms to standard modal axioms. This correspondence (as well as many other such correspondences between certain modal logics and justification logics) can be shown in a formal way. While it is easy to see, that replacing all justification terms by $\square$ in a theorem of LP yields a theorem of S4, the other direction is much more involved and known as the realization

\footnotetext{
^ Supported by Swiss National Science Foundation grant 200021-117699.

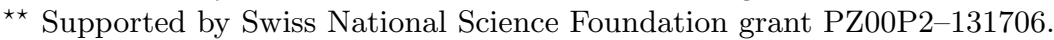




\begin{tabular}{llr}
\multicolumn{1}{c}{ S4 axioms } & \multicolumn{1}{c}{ LP axioms } & \\
$\square(A \rightarrow B) \rightarrow(\square A \rightarrow \square B)$ & $t:(A \rightarrow B) \rightarrow(s: A \rightarrow t \cdot s: B)$ & (application) \\
$\square A \rightarrow A$ & $t: A \rightarrow A$ & (reflexivity) \\
$\square A \rightarrow \square \square A$ & $t: A \rightarrow ! t: t: A$ & (inspection) \\
& $t: A \vee s: A \rightarrow t+s: A$ & (sum)
\end{tabular}

Fig. 1. Non-propositional axioms of S4 and LP

theorem. See [7] for a uniform proof and survey of realization theorems for all logics in the modal cube.

Fitting [11] introduced epistemic semantics for justification logics. The socalled Fitting models are Kripke models $(W, R, \nu)$ augmented by an evidence relation $\mathcal{E}$ that states which terms are admissible evidence for which formulae.

Filtrations are a tool in modal logic for obtaining from a given, usually infinite, model a smaller, usually finite, model by factoring the set of worlds with respect to a certain equivalence relation. As noted in [6], filtrations were first introduced in [19] and given their name in [15]. Given the close relationship between Fitting models and Kripke models, it is a natural task to adopt filtrations for justification logics. The crucial step is of course to take into account the evidence relation when identifying states.

Filtrations are often used to prove a finite model property and thereby establish decidability of a given modal logic, see e.g. [6]. Decidability for the justification logics presented here was originally shown in $[12,13,16]$. We adapt the filtration technique from modal logic to obtain an alternative uniform proof of decidability for these justification logics. We then apply the newly developed technique to establish the decidability of the multi-agent justification logic with common knowledge presented in [9].

In Section 2, we introduce the syntax and semantics of the justification logics we are using. In Section 3, we define filtrations for justification logics and prove their basic properties. We treat two specific examples of filtrations in Sections 4 and 5. In Section 6, we use these two examples to prove the decidability of the defined justification logics. This also leads us to investigate general properties necessary for the decidability of justification logics and enables us to prove the decidability of a multi-agent justification logic with common knowledge in Section 7 .

\section{Justification Logics}

Justification terms are built from constants $c_{i}$ and variables $x_{i}$ according to the following grammar:

$$
t::=c_{i}\left|x_{i}\right|(t \cdot t)|(t+t)| ! t \quad .
$$

We denote the set of terms by Tm. Formulae are built from atomic propositions $p_{i}$ according to the following grammar:

$$
F::=p_{i}|\neg F|(F \rightarrow F) \mid t: F \quad .
$$


Prop denotes the set of atomic propositions and Fm denotes the set of formulae.

The set $\operatorname{Sub}(F)$ of subformulae of a given formula $F$ is defined inductively as follows

$$
\begin{aligned}
& -\operatorname{Sub}\left(p_{i}\right):=\left\{p_{i}\right\} \\
& -\operatorname{Sub}(\neg F):=\{\neg F\} \cup \operatorname{Sub}(F) \\
& -\operatorname{Sub}\left(F_{1} \rightarrow F_{2}\right):=\left\{F_{1} \rightarrow F_{2}\right\} \cup \operatorname{Sub}\left(F_{1}\right) \cup \operatorname{Sub}\left(F_{2}\right) \\
& -\operatorname{Sub}(t: F):=\{t: F\} \cup \operatorname{Sub}(F)
\end{aligned}
$$

A set of formulae $\Phi \subseteq$ Fm is closed under subformulae if $\bigcup_{F \in \Phi} \operatorname{Sub}(F) \subseteq \Phi$.

The axioms of $J_{C S}$ consist of all instances of the following schemes:

A1 finitely many schemes axiomatizing classical propositional logic

A2 $t:(A \rightarrow B) \rightarrow(s: A \rightarrow t \cdot s: B)$

A3 $t: A \vee s: A \rightarrow t+s: A$

We will consider extension of $\mathrm{J}_{\mathrm{CS}}$ by the following axioms schemes.

(jd) $t: \perp \rightarrow \perp$

(jt) $t: A \rightarrow A$

(j4) $t: A \rightarrow ! t: t: A$

A constant specification CS for a logic L is any subset

$$
\mathrm{CS} \subseteq\{c: A \mid c \text { is a constant and } A \text { is an axiom of } \mathrm{L}\} .
$$

A constant specification CS for a logic $L$ is called

1. axiomatically appropriate if for each axiom $A$ of $\mathrm{L}_{\mathrm{CS}}$ there is a constant $c$ such that $c: A \in \mathrm{CS}$

2. schematic if for each constant $c$ the set $\{A \mid c: A \in \mathrm{CS}\}$ consists of one or several (possibly zero) axiom schemes, i.e., every constant justifies certain axiom schemes.

For a constant specification CS the deductive system $J_{C S}$ is the Hilbert system given by the axioms $\mathrm{A} 1-\mathrm{A} 3$ and by the rules modus ponens and axiom necessitation:

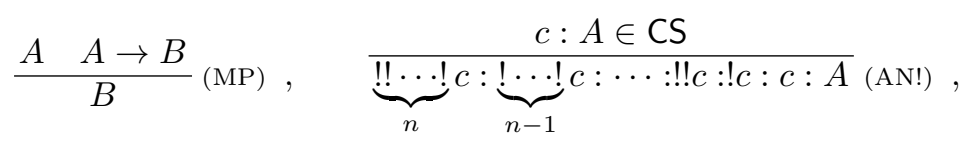

where $n \geq 0$. In the presence of the $\mathrm{j} 4$ axiom a simplified axiom necessitation rule can be used:

$$
\frac{c: A \in \mathrm{CS}}{c: A}(\mathrm{AN})
$$

Table 1 defines the various logics we consider.

We now present the semantics for these logics 


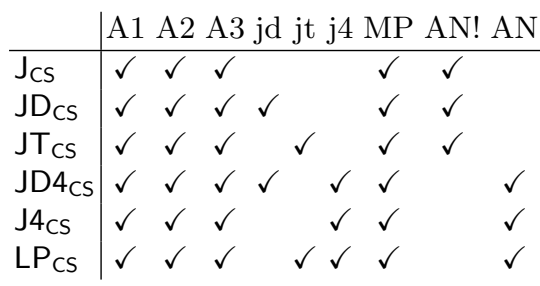

Table 1. Deductive Systems

Definition 1 (Evidence relation). Let $(W, R)$ be a Kripke frame, i.e., $W \neq \varnothing$ and $R \subseteq W \times W$, and CS be a constant specification. An admissible evidence relation $\mathcal{E}$ for a logic $\mathrm{L}_{\mathrm{CS}}$ is a subset of $\mathrm{Tm} \times \mathrm{Fm} \times W$ that satisfies the closure conditions:

1. if $(s, A, w) \in \mathcal{E}$ or $(t, A, w) \in \mathcal{E}$, then $(s+t, A, w) \in \mathcal{E}$

2. if $(s, A \rightarrow B, w) \in \mathcal{E}$ and $(t, A, w) \in \mathcal{E}$, then $(s \cdot t, B, w) \in \mathcal{E}$

Depending on whether or not the logic $\mathrm{L}_{\mathrm{CS}}$ contains the $j 4$ axiom, the evidence function has to satisfy one of the following two sets of closure conditions. If $\mathrm{L}_{\mathrm{CS}}$ does not include the $j_{4}$ axiom, then the additional requirement is:

3. if $c: A \in \mathrm{CS}$ and $w \in W$, then $(\underbrace{! ! \ldots !}_{n} c, \underbrace{! \ldots !}_{n-1} c: \cdots: ! ! c: ! c: c: A, w) \in \mathcal{E}$

If $\mathrm{L}_{\mathrm{CS}}$ includes the $j 4$ axiom, then the additional requirement is:

4. if $c: A \in \mathrm{CS}$ and $w \in W$, then $(c, A, w) \in \mathcal{E}$

5. if $(t, A, w) \in \mathcal{E}$, then $(! t, t: A, w) \in \mathcal{E}$

6. if $(t, A, w) \in \mathcal{E}$ and $w R v$, then $(t, A, v) \in \mathcal{E}$

If we drop condition 6 , then we say $\mathcal{E}$ is a t-evidence relation.

Sometimes we use $\mathcal{E}(s, A, w)$ for $(s, A, w) \in \mathcal{E}$.

\section{Definition 2 (Evidence bases).}

1. An evidence base $\mathcal{B}$ is a subset of $T m \times F m \times W$.

2. An evidence relation $\mathcal{E}$ is based on $\mathcal{B}$, if $\mathcal{B} \subseteq \mathcal{E}$.

The closure conditions in the definition of admissible evidence function give rise to a monotone operator. The minimal evidence relation based on $\mathcal{B}$ is the least fixed point of that operator and thus always exists.

Definition 3 (Model). Let CS be a constant specification. A Fitting model for a logic $\mathrm{L}_{\mathrm{CS}}$ is a quadruple $\mathcal{M}=(W, R, \mathcal{E}, \nu)$ where

- $(W, R)$ is a Kripke frame such that

- if $\mathrm{L}_{\mathrm{CS}}$ includes the $j_{4}$ axiom, then $R$ is transitive;

- if $\mathrm{L}_{\mathrm{CS}}$ includes the jt axiom, then $R$ is reflexive; 
- if $\mathrm{L}_{\mathrm{CS}}$ includes the $j d$ axiom, then $R$ is serial.

$-\mathcal{E}$ is an admissible evidence relation for $\mathrm{L}_{\mathrm{CS}}$ over the frame $(W, R)$,

$-\nu:$ Prop $\rightarrow \mathcal{P}(W)$, called a valuation function.

Definition 4 (Satisfaction relation). The relation of formula $A$ being satisfied in a model $\mathcal{M}=(W, R, \mathcal{E}, \nu)$ at a world $w \in W$ is defined by induction on the structure of $A$ by

$-\mathcal{M}, w \Vdash p_{i}$ if and only if $w \in \nu\left(p_{i}\right)$

$-\Vdash$ commutes with Boolean connectives

$-\mathcal{M}, w \Vdash t: B$ if and only if

1) $\mathcal{M}, v \Vdash B$ for all $v \in W$ with $w R v$ and

2) $(t, B, w) \in \mathcal{E}$

We say a formula $A$ is valid in a model $\mathcal{M}=(W, R, \mathcal{E}, \nu)$ if for all $w \in W$ we have $\mathcal{M}, w \Vdash A$. We say a formula $A$ is valid for a logic $\mathrm{L}_{\mathrm{CS}}$ if for all models $\mathcal{M}$ for $\mathrm{L}_{\mathrm{CS}}$ we have that $A$ is valid in $\mathcal{M}$.

The logics defined above are sound and complete (with a restriction in case of the logics containing the jd axiom). See $[3,11,17]$ for the full proofs of the following results.

Soundness can be obtained by an easy induction on the derivation of the formula.

Theorem 5 (Soundness). Let CS be a constant specification. If a formula $A$ is derivable in a logic $\mathrm{L}_{\mathrm{CS}}$, then $A$ is valid for $\mathrm{L}_{\mathrm{CS}}$.

For completeness a canonical model construction is used. The axiomatical appropriateness of the constant specification in case the logic contains the $\mathrm{jd}$ axiom is necessary to show the seriality condition on the accessibility relation.

\section{Theorem 6 (Completeness).}

1. Let CS be a constant specification. If a formula $A$ is not derivable in $\mathrm{L}_{\mathrm{CS}} \in$ $\left\{\mathrm{J}_{\mathrm{CS}}, \mathrm{JT}_{\mathrm{CS}}, \mathrm{J}_{\mathrm{CS}}, \mathrm{LP}_{\mathrm{CS}}\right\}$, then there exists a model $\mathcal{M}$ for $\mathrm{L}_{\mathrm{CS}}$ with $\mathcal{M}, w \forall A$ for some world $w$ in $\mathcal{M}$.

2. Let CS be an axiomatically appropriate constant specification. If a formula $A$ is not derivable in $\mathrm{L}_{\mathrm{CS}} \in\left\{\mathrm{JD}_{\mathrm{CS}}, \mathrm{JD}_{\mathrm{CS}}\right\}$, then there exists a model $\mathcal{M}$ for $\mathrm{L}_{\mathrm{CS}}$ with $\mathcal{M}, w \|$ A for some world $w$ in $\mathcal{M}$.

\section{Filtrations}

Given the close relationship of models for justification logics to Kripke models, it is not surprising that the two definitions of filtrations look very similar. The major difference is that we have to take the evidence relation into consideration. In modal logic we identify worlds that behave the same way, whereas in justification logic we identify worlds that behave the same way for the same reason. 
Definition 7 (Filtration). Let $\mathcal{M}=(W, R, \mathcal{E}, \nu)$ be a model and $\Phi$ some set of formulae that is closed under subformulae. We define an equivalence relation $=_{\Phi}$ on $W$ by setting $w=_{\Phi} v$ if and only if for all $A \in \Phi$

$$
M, w \Vdash A \text { if and only if } M, v \Vdash A
$$

and for all $t: B \in \Phi$

$$
\mathcal{E}(t, B, w) \text { if and only if } \mathcal{E}(t, B, v) .
$$

We denote the equivalence classes of $\Phi$ by $[w]_{\Phi}$. When $\Phi$ is clear from the context, we will often only write $[w]$ instead of $[w]_{\Phi}$.

A model $\mathcal{M}_{\Phi}=\left(W_{\Phi}, R_{\Phi}, \mathcal{E}_{\Phi}, \nu_{\Phi}\right)$ is called a filtration of $\mathcal{M}$ through $\Phi$ if it satisfies the following:

1. $W_{\Phi}=\left\{[w]_{\Phi} \mid w \in W\right\}$

2. $R_{\Phi}$ satisfies

(R1) for all $w, v \in W$ if $R(w, v)$, then $R_{\Phi}\left([w]_{\Phi},[v]_{\Phi}\right)$

(R2) for all $[w]_{\Phi},[v]_{\Phi} \in W_{\Phi}$, if $R_{\Phi}\left([w]_{\Phi},[v]_{\Phi}\right)$, then for any $t: B \in \Phi$ we have

$$
\text { if } \mathcal{M}, w \Vdash t: B \text { then } \mathcal{M}, v \Vdash B
$$

3. $\mathcal{E}_{\Phi}$ satisfies

(E1) for all $w \in W$ and $t: B \in \Phi$ we have

$$
\text { if } \mathcal{M}, w \Vdash t: B \text { then }\left(t, B,[w]_{\Phi}\right) \in \mathcal{E}_{\Phi}
$$

(E2) for all $w \in W$ and $t: B \in \Phi$ we have

$$
\text { if }\left(t, B,[w]_{\Phi}\right) \in \mathcal{E}_{\Phi} \text { then }(t, B, w) \in \mathcal{E}
$$

4. $\nu_{\Phi}$ satisfies for all atomic propositions $p \in \Phi$

$$
\nu_{\Phi}(p)=\left\{[w]_{\Phi} \mid w \in \nu(p)\right\}
$$

There are two major changes of the definition compared to the case for modal logic. The first change concerns the definition of the equivalence relation to identify worlds. Whereas a modal formula $\square B$ can only fail due to the existence of an accessible world not satisfying $B$, a justification formula $t: B$ might fail in two ways: either $B$ is not satisfied in an accessible world or $t$ is not admissible evidence for $B$ at the current world. So we have to refine our equivalence relation to only identify worlds that do not only satisfy the same formulae but also behave the same with respect to the evidence relation. The second change concerns the evidence relation of the filtration: it has to satisfy conditions similar to the Minand Max-conditions (R1) and (R2) for the accessibility relation.

The crucial property of a filtration of a model through $\Phi$ is that the behavior of the model and the filtration is the same with respect to formulae in $\Phi$ : 
Lemma 8. Let $\mathcal{M}=(W, R, \mathcal{E}, \nu)$ be a model, $\Phi$ a set of formulae closed under subformulae, and $\mathcal{M}_{\Phi}=\left(W_{\Phi}, R_{\Phi}, \mathcal{E}_{\Phi}, \nu_{\Phi}\right)$ a filtration of $\mathcal{M}$ through $\Phi$. Then for all worlds $w \in W$ and formulae $A \in \Phi$ we have

$$
\mathcal{M}_{\Phi},[w]_{\Phi} \Vdash A \text { if and only if } \mathcal{M}, w \Vdash A \text {. }
$$

Proof. The proof is by induction on the structure of $A$. The case for propositional variables is immediate by the definition of $\nu_{\Phi}$ and the cases for the propositional connectives are immediate by the induction hypothesis. Let us now consider the case $A=t: B$.

First we show the direction from right to left. Assume $\mathcal{M}, w \Vdash t: B$. If $R_{\Phi}\left([w]_{\Phi},[v]_{\Phi}\right)$, then by $(\mathrm{R} 2)$ we have $\mathcal{M}, v \Vdash B$. By the induction hypothesis we get $\mathcal{M}_{\Phi},[v]_{\Phi} \Vdash B$. Further from (E1) we get $\left(t, B,[w]_{\Phi}\right) \in \mathcal{E}_{\Phi}$ and thus $\mathcal{M}_{\Phi},[w]_{\Phi} \Vdash t: B$.

For the other direction suppose $\mathcal{M}_{\Phi},[w]_{\Phi} \Vdash t: B$, that is

$$
\begin{gathered}
\mathcal{M}_{\Phi},[v]_{\Phi} \Vdash B \text { for all }[v]_{\Phi} \text { with } R_{\Phi}\left([w]_{\Phi},[v]_{\Phi}\right) \\
\qquad\left(t, B,[w]_{\Phi}\right) \in \mathcal{E}_{\Phi}
\end{gathered}
$$

If $R(w, v)$, then by (R1) also $R_{\Phi}\left([w]_{\Phi},[v]_{\Phi}\right)$ and by (1) and the induction hypothesis we get $\mathcal{M}, v \Vdash B$. Furthermore, from (2) and (E2) we get $\mathcal{E}(t, B, w)$ and we conclude $\mathcal{M}, w \Vdash t: B$.

A filtration inherits some conditions on the accessibility relations. Furthermore, a filtration through a finite set has finitely many worlds.

Lemma 9. Let $\mathcal{M}=(W, R, \mathcal{E}, \nu)$ be a model, $\Phi$ a set of formulae closed under subformulae, and $\mathcal{M}_{\Phi}=\left(W_{\Phi}, R_{\Phi}, \mathcal{E}_{\Phi}, \nu_{\Phi}\right)$ a filtration of $\mathcal{M}$ through $\Phi$.

1. If $R$ is serial, so is $R_{\Phi}$.

2. If $R$ is reflexive, so is $R_{\Phi}$.

3. If $\Phi$ is finite, then so is $W_{\Phi}$.

Proof. The first two claims follow immediately from (R1). The last claim follows from the fact that each element $[w]_{\Phi} \in W_{\Phi}$ can be characterized by the set of formulae $A \in \Phi$ that hold in $[w]_{\Phi}$ as well as the set of formulae $t: B \in \Phi$ with $\mathcal{E}_{\Phi}\left(t, B,[w]_{\Phi}\right)$ and the fact that $\mathcal{P}(\Phi) \times \mathcal{P}(\Phi)$ has only finitely many elements.

\section{Non-transitive Case}

As a first example we will define filtrations for logics not containing the $\mathrm{j} 4 \mathrm{axiom}$.

Definition 10 (Filtration: non-transitive case). Let $\mathcal{M}=(W, R, \mathcal{E}, \nu)$ be a model and $\Phi$ a set of formulae closed under subformulae. We consider the filtration $\mathcal{M}_{\Phi}^{n t}=\left(W_{\Phi}^{n t}, R_{\Phi}^{n t}, \mathcal{E}_{\Phi}^{n t}, \nu_{\Phi}^{n t}\right)$ that is given by

1. $W_{\Phi}^{\text {nt }}$ is the set of equivalence classes induced by $=_{\Phi}$ 
2. $R_{\Phi}^{n t}([w],[v])$ if and only if for all $t: B \in \Phi$ we have $\mathcal{M}, w \Vdash t: B$ implies $\mathcal{M}, v \Vdash B$

3. $\mathcal{E}_{\Phi}^{n t}$ is the minimal evidence relation based on $\mathcal{B}_{\Phi}^{n t}$, where

$$
\mathcal{B}_{\Phi}^{\text {nt }}(t, B,[v]) \text { if and only if } t: B \in \Phi \text { and } \mathcal{E}(t, B, v) .
$$

4. $\nu_{\Phi}^{n t}$ is given by

$$
\nu_{\Phi}^{n t}(p)= \begin{cases}\{[w] \mid w \in \nu(p)\} & \text { if } p \in \Phi, \\ \emptyset & \text { otherwise. }\end{cases}
$$

Lemma 11. $\mathcal{M}_{\Phi}^{\text {nt }}$ is a filtration of $\mathcal{M}$ through $\Phi$.

Proof. We have to check the following conditions.

(R1) Assume $R(w, v)$. If $\mathcal{M}, w \Vdash t: B$, then $\mathcal{M}, v \Vdash B$. Thus we conclude $R_{\Phi}^{\mathrm{nt}}([w],[v])$.

(R2) Let $t: B \in \Phi$ and $R_{\Phi}^{\text {nt }}([w],[v])$. If $\mathcal{M}, w \Vdash t: B$, then we get $\mathcal{M}, v \Vdash B$ immediately from the definition of $R_{\Phi}^{\text {nt }}$.

(E1) Assume $t: B \in \Phi$ and $\mathcal{M}, w \Vdash t: B$. We have $\mathcal{E}(t, B, w)$ and we immediately get $\mathcal{E}_{\Phi}^{\text {nt }}(t, B,[w])$ by the definition of $\mathcal{E}_{\Phi}^{\text {nt }}$.

(E2) We show for all $t: B$, not only for those contained in $\Phi$, that for all $w^{\prime} \in[w]$

$$
\mathcal{E}_{\Phi}^{\text {nt }}(t, B,[w]) \text { implies } \mathcal{E}\left(t, B, w^{\prime}\right) .
$$

We proceed by induction on the construction of $\mathcal{E}_{\Phi}^{\text {nt }}$.

- If $\mathcal{E}_{\Phi}^{\text {nt }}(t, B,[w])$ because $\mathcal{B}_{\Phi}^{\text {nt }}(t, B,[w])$, then by definition of $\mathcal{B}_{\Phi}^{\text {nt }}$ we have that $t: B \in \Phi$ and $\mathcal{E}\left(t, B, w^{\prime \prime}\right)$ for some $w^{\prime \prime} \in[w]$. By $w^{\prime}=_{\Phi} w^{\prime \prime}$ we conclude $\mathcal{E}\left(t, B, w^{\prime}\right)$.

- If $t=t_{1}+t_{2}$ and $\mathcal{E}_{\Phi}^{\text {nt }}(t, B,[w])$ because of $\mathcal{E}_{\Phi}^{\text {nt }}\left(t_{i}, B,[w]\right)$ (for some $i \in$ $\{1,2\})$, then by induction hypothesis we get that $\mathcal{E}\left(t_{i}, B, w^{\prime}\right)$ and thus also $\mathcal{E}\left(t_{1}+t_{2}, B, w^{\prime}\right)$ by the closure conditions on $\mathcal{E}$.

- If $t=t_{1} \cdot t_{2}$ and $\mathcal{E}_{\Phi}^{\mathrm{nt}}(t, B,[w])$ because there is an $A \in \mathrm{Fm}$ such that $\mathcal{E}_{\Phi}^{\text {nt }}\left(t_{1}, A \rightarrow B,[w]\right)$ and $\mathcal{E}_{\Phi}^{\text {nt }}\left(t_{2}, A,[w]\right)$, then by induction hypothesis $\mathcal{E}\left(t_{1}, A \rightarrow B, w^{\prime}\right)$ and $\mathcal{E}\left(t_{2}, A, w^{\prime}\right)$. So, by the closure conditions, we get $\mathcal{E}\left(t_{1} \cdot t_{2}, B, w^{\prime}\right)$.

- The case for axiom necessitation is trivial, as we have

$$
\mathcal{E}(\underbrace{! \cdots ! !}_{n} c, \underbrace{! \cdots ! !}_{n-1} c: \cdots: ! c: c: A, v)
$$

for any world $v \in W$.

\section{Transitive Case}

The case for logics containing the $\mathrm{j} 4$ axiom is a bit more involved, as now the accessibility relation of the filtration has to be transitive, which is not guaranteed by the definition of filtration. 
Definition 12 (Filtration: transitive case). Let $\mathcal{M}=(W, R, \mathcal{E}, \nu)$ be a model and $\Phi$ a set of formulae closed under subformulae. We consider the filtration $\mathcal{M}_{\Phi}^{\text {tr }}=\left(W_{\Phi}^{\text {tr }}, R_{\Phi}^{\text {tr }}, \mathcal{E}_{\Phi}^{\text {tr }}, \nu_{\Phi}^{\text {tr }}\right)$ that is given by

1. $W_{\Phi}^{\text {tr }}$ is the set of equivalence classes induced by $=_{\Phi}$

2. $R_{\Phi}^{t r}([w],[v])$ if and only if for all $t: B \in \Phi$ we have $\mathcal{M}, w \Vdash t: B$ implies $\mathcal{M}, v \Vdash B \wedge t: B$

3. $\mathcal{E}_{\Phi}^{\text {tr }}$ is the minimal t-evidence relation based on $\mathcal{B}_{\Phi}^{\text {tr }}$, where

$$
\mathcal{B}_{\Phi}^{\text {tr }}(t, B,[v]) \text { if and only if } t: B \in \Phi \text { and } \mathcal{M}, v \Vdash t: B \text {. }
$$

4. $\nu_{\Phi}^{\text {tr }}$ is given by

$$
\nu_{\Phi}^{\operatorname{tr}}(p)= \begin{cases}\{[w] \mid w \in \nu(p)\} & \text { if } p \in \Phi, \\ \emptyset & \text { otherwise. }\end{cases}
$$

As a first step we have to show that $\mathcal{E}_{\Phi}^{\text {tr }}$ as defined is not only a t-evidence relation but an actual evidence relation.

Lemma 13. $\mathcal{E}_{\Phi}^{\text {tr }}$ is an admissible evidence relation over $\left(W_{\Phi}^{t r}, R_{\Phi}^{\text {tr }}\right)$.

Proof. We have to show that condition (6) in Definition 1 holds, i.e., we have to show

$$
\mathcal{E}_{\Phi}^{\mathrm{tr}}(t, B,[w]) \text { and } R_{\Phi}^{\mathrm{tr}}([w],[v]) \text { imply } \mathcal{E}_{\Phi}^{\mathrm{tr}}(t, B,[v])
$$

So assume $\mathcal{E}_{\Phi}^{\mathrm{tr}}(t, B,[w])$ and $R_{\Phi}^{\mathrm{tr}}([w],[v])$. We now show $\mathcal{E}_{\Phi}^{\mathrm{tr}}(t, B,[v])$ by induction on the construction of $\mathcal{E}_{\Phi}^{\text {tr }}$.

Let $\mathcal{E}_{\Phi}^{\text {tr }}(t, B,[w])$ because of $\mathcal{B}_{\Phi}^{\text {tr }}(t, B,[w])$. We have $t: B \in \Phi$ and $\mathcal{M}, w \Vdash t: B$ by definition of $\mathcal{B}_{\Phi}^{\text {tr }}$. Since $R_{\Phi}([w],[v])$, it follows that $\mathcal{M}, v \Vdash B \wedge t: B$ and, in particular, $\mathcal{M}, v \Vdash t: B$. Thus, $\mathcal{B}_{\Phi}^{\text {tr }}(t, B,[v])$ by definition of $\mathcal{B}_{\Phi}^{\text {tr }}$, and clearly $\mathcal{E}_{\Phi}^{\mathrm{tr}}(t, B,[v])$.

Let us now distinguish the different possible closure conditions from Definition 1:

1. Assume we have $t=t_{1}+t_{2}$ and $\mathcal{E}_{\Phi}^{\mathrm{tr}}(t, B,[w])$ because of $\mathcal{E}_{\Phi}^{\mathrm{tr}}\left(t_{i}, B,[w]\right)$ for $i=1$ or $i=2$. Then by induction hypothesis $\mathcal{E}_{\Phi}^{\mathrm{tr}}\left(t_{i}, B,[v]\right)$ and thus also $\mathcal{E}_{\Phi}^{\mathrm{tr}}(t, B,[v])$.

2. The case for - and ! follows immediately from the induction hypothesis in the same manner as the previous case.

3. The case for axiom necessitation (AN) trivially holds.

The accessibility relation for the filtration is transitive.

Lemma 14. $R_{\Phi}^{\text {tr }}$ is transitive.

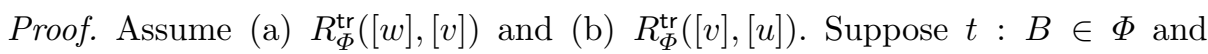
$\mathcal{M}, w \Vdash t: B$. By (a) we get $\mathcal{M}, v \Vdash t: B$. Then by (b) we get $\mathcal{M}, u \Vdash B \wedge t: B$. Hence, we conclude $R_{\Phi}^{\mathrm{tr}}([w],[u])$. 
Lemma 15. $\mathcal{M}_{\Phi}^{\text {tr }}$ is a filtration of $\mathcal{M}$ through $\Phi$.

Proof. We have to check the following conditions.

(R1) Assume $R(w, v)$. If $\mathcal{M}, w \Vdash t: B$, then $\mathcal{M}, v \Vdash B$ and $\mathcal{M}, w \Vdash ! t: t: B$ which implies $\mathcal{M}, v \Vdash t: B$. Thus we conclude $R_{\Phi}^{\mathrm{tr}}([w],[v])$.

(R2) Let $t: B \in \Phi$ and $R_{\Phi}([w],[v])$. If $\mathcal{M}, w \Vdash t: B$, then we get $\mathcal{M}, v \Vdash B$ immediately from the definition of $R_{\Phi}^{\mathrm{tr}}$.

(E1) Assume $t: B \in \Phi$ and $\mathcal{M}, w \Vdash t: B$. We immediately get $\mathcal{E}_{\Phi}^{\mathrm{tr}}(t, B,[w])$ by the definition of $\mathcal{E}_{\Phi}^{\text {tr }}$.

(E2) As in the proof of Lemma 11 we can show for all $t: B$ and all $w^{\prime} \in[w]$

$$
\mathcal{E}_{\Phi}^{\mathrm{tr}}(t, B,[w]) \text { implies } \mathcal{E}\left(t, B, w^{\prime}\right)
$$

\section{Decidability}

The theorems in this section originate from [13]. We thus only give proof sketches for the sake of brevity.

Definition 16 (Finitary model). A model $\mathcal{M}=(W, R, \mathcal{E}, \nu)$ is called finitary if

1. $W$ is finite,

2. there exists a finite base $\mathcal{B}$ such that $\mathcal{E}$ is the minimal evidence relation based on $\mathcal{B}$, and

3. the set $\{(w, p) \in W \times \operatorname{Prop} \mid w \in \nu(p)\}$ is finite.

Using filtrations we see that if a formula is satisfiable, then it is satisfiable in a finitary model. Thus we have the following

\section{Lemma 17 (Completeness w.r.t. finitary models).}

1. Let $\mathrm{L}_{\mathrm{CS}} \in\left\{\mathrm{J}_{\mathrm{CS}}, \mathrm{JT}_{\mathrm{CS}}, \mathrm{J}_{\mathrm{CS}}, \mathrm{LP}_{\mathrm{CS}}\right\}$ and $\mathrm{CS}$ be a constant specification for $\mathrm{L}$. If a formula $A$ is not derivable in $\mathrm{L}_{\mathrm{CS}}$, then there exists a finitary model $\mathcal{M}$ for $\mathrm{L}_{\mathrm{CS}}$ with $\mathcal{M}, w \forall A$ for some world $w$ in $\mathcal{M}$.

2. Let $\mathrm{L}_{\mathrm{CS}} \in\left\{\mathrm{JD}_{\mathrm{CS}}, \mathrm{JD}_{\mathrm{CS}}\right\}$ and $\mathrm{CS}$ be an axiomatically appropriate constant specification for $\mathrm{L}$. If a formula $A$ is not derivable in $\mathrm{L}_{\mathrm{CS}}$, then there exists a finitary model $\mathcal{M}$ for $\mathrm{L}_{\mathrm{CS}}$ with $\mathcal{M}, w \forall A$ for some world $w$ in $\mathcal{M}$.

Proof. Let CS be as required above. If $A$ is not derivable in $\mathrm{L}_{\mathrm{CS}}$, then by Theorem 6 there exists a model $\mathcal{M}$ for $\mathrm{L}_{\mathrm{CS}}$ with $\mathcal{M}, v \forall \forall$ for some world $v$ in $\mathcal{M}$. Now set $\Phi:=\operatorname{Sub}(A)$ and let $\mathcal{M}_{\Phi}$ denote either $\mathcal{M}_{\Phi}^{\text {nt }}$ or $\mathcal{M}_{\Phi}^{\text {tr }}$ from Definitions 10 and 12 respectively, depending on whether $\mathrm{L}_{\mathrm{CS}}$ contains the $\mathrm{j} 4$ axiom. It is easy to see that $\mathcal{M}_{\Phi}$ is a finitary model: by Lemma 9 the set of worlds is finite and, by definition of $\mathcal{M}_{\Phi}$, the evidence relation is finitely based and the valuation function satisfies condition 3 from Definition 16 . Finally, since $\mathcal{M}_{\Phi}$ is a filtration of $\mathcal{M}$ through $\Phi$ by Lemma 11 or by Lemma 15 , by Lemma 8 we have $\mathcal{M}_{\Phi},[v] \forall A$. 
Corollary 18. All statements of Lemma 17 hold if an additional restriction is imposed that the domain of the model $\mathcal{M}$ be a finite subset of $\mathbb{N}$.

Proof. The claim follows trivially from Lemma 17 by renaming worlds to natural numbers.

The following theorem is a simple instance of Post's theorem [18]: A set is decidable if and only if both the set and its complement are recursively enumerable.

Theorem 19. A logic is decidable if it is recursively enumerable and is sound and complete with respect to a set $\mathcal{C}$ such that

1. $\mathcal{C}$ is a recursively enumerable set of finite models and

2. the relation $\mathcal{M}, w \Vdash A$ between models $\mathcal{M} \in \mathcal{C}$, worlds $w$ in $\mathcal{M}$, and formulae $A$ is decidable.

Proof. We give a proof sketch, for full details cf. [13, Theorem 4.3.3]

Given a formula $A$, we can simultaneously enumerate theorems $B_{0}, B_{1}, \ldots$ of the logic and potential counter-models $\mathcal{M}_{0}, \mathcal{M}_{1}, \ldots \in \mathcal{C}$ and at each step check whether (a) $A=B_{i}$ or (b) $\mathcal{M}_{i}, w \forall A$ for some $w \in \mathcal{M}_{i}$. Eventually either (a) or (b) will hold for some $i$, thus indicating whether the logic proves $A$.

Lemma 20. Let $\mathrm{L}_{\mathrm{CS}} \in\left\{\mathrm{J}_{\mathrm{CS}}, \mathrm{J} \mathrm{D}_{\mathrm{CS}}, \mathrm{JD} 4_{\mathrm{CS}}, \mathrm{JT} \mathrm{CS}_{\mathrm{CS}}, \mathrm{J} 4_{\mathrm{CS}}, \mathrm{LP}_{\mathrm{CS}}\right\}$. The set of finitary models for $\mathrm{L}_{\mathrm{CS}}$ with the domain being a finite subset of $\mathbb{N}$ is recursively enumerable.

Proof. We give a proof sketch, for full details cf. [13, Lemma 4.4.6].

It is obvious that the set of such models for $\mathrm{J}_{\mathrm{CS}}$ can be recursively enumerated. Models of each of the other five logics must additionally satisfy certain conditions on the accessibility relation, some combination of transitivity, reflexivity, and seriality. Since each of these conditions can be effectively verified, the models of $J_{C S}$ that are unsuitable for a given logic can be effectively removed from the enumeration of models for $L_{C S}$.

Lemma 21. Let $\mathrm{CS}$ be a decidable schematic constant specification and $\mathrm{L}_{\mathrm{CS}} \in$ $\left\{\mathrm{J}_{\mathrm{CS}}, \mathrm{JD}_{\mathrm{CS}}, \mathrm{JD} 4_{\mathrm{CS}}, \mathrm{JT}_{\mathrm{CS}}, \mathrm{J}_{\mathrm{CS}}, \mathrm{LP}_{\mathrm{CS}}\right\}$. Let $\mathcal{M}=(W, R, \mathcal{E}, \nu)$ be a finitary model for $\mathrm{L}_{\mathrm{CS}}$. Then the relation $\mathcal{M}, w \Vdash A$ between worlds $w \in W$ and formulae $A$ is decidable.

Proof. We give a proof sketch, for full details cf. [13, Corollary 4.4.8].

We can show this by induction on the formula $A$, the cases for propositions and Boolean connectives being trivial.

The crucial step is to show that the relation $\mathcal{E}(t, B, w)$ between terms $t \in \mathrm{Tm}$, formulae $B \in \mathrm{Fm}$ and worlds $w \in W$ is decidable (see [13, Lemma 4.4.7]).

Let $\mathcal{B}$ be the base for the minimal evidence relation $\mathcal{E}$ of $\mathcal{M}$. Given a fixed term $t$, we will construct a sequence of sets $\mathcal{E}_{t}^{i}(w)$ inductively, which can be seen as a partial evidence function that lists all formulae for which $t$ or one of its subterms are admissible evidence at world $w$. 
In order to keep the sets finite and as we are given a schematic constant specification, we will use variables $X, Y, \ldots$ ranging over schemes of formulae and variables $P, Q, \ldots$ ranging over formulae. Also, we assume that our constant specification is given in terms of schemes, i.e.

$$
\mathrm{CS}=\{c: X \mid c \text { is a constant and } X \text { is a scheme }\} \text {. }
$$

The sets are defined as follows

$$
\begin{aligned}
\mathcal{E}_{t}^{0}(w):=\{(s, B) \mid \mathcal{B}(s, B, w) \text { and } s & \in \operatorname{Sub}(t)\} \\
& \cup\{(c, X) \mid c: X \in \mathrm{CS} \text { and } c \in \operatorname{Sub}(t)\}
\end{aligned}
$$

Assume $\mathcal{E}_{t}^{n}(w)$ has been constructed, in order to obtain $\mathcal{E}_{t}^{n+1}(w)$ add the following

- $\left(s_{1} \cdot s_{2}, Y_{1} \sigma\right)$ for any $\left(s_{1}, X_{1} \rightarrow Y_{1}\right) \in \mathcal{E}_{t}^{n}(w)$ and $\left(s_{2}, X_{2}\right) \in \mathcal{E}_{t}^{n}(w)$ such that the most general unifier $\sigma$ of $X_{1}$ and $X_{2}$ exists and $s_{1} \cdot s_{2} \in \operatorname{Sub}(t)$

$-\left(s_{1} \cdot s_{2}, Q\right)$ for any $\left(s_{1}, P\right) \in \mathcal{E}_{t}^{n}(w)$ and $\left(s_{2}, X_{2}\right) \in \mathcal{E}_{t}^{n}(w)$ where $Q$ is a fresh variable over formulas and $s_{1} \cdot s_{2} \in \operatorname{Sub}(t)$

$-\left(s_{1}+s_{2}, X\right)$ for any $\left(s_{1}, X\right)$ or $\left(s_{2}, X\right) \in \mathcal{E}_{t}^{n}(w)$ with $s_{1}+s_{2} \in \operatorname{Sub}(t)$

- depending on whether the logic $\mathrm{L}_{\mathrm{CS}}$ contains the $\mathrm{j} 4$ axiom, we distinguish the following two cases: If the logic does not contain the $\mathrm{j} 4$ axiom, we add

- $(\underbrace{! ! \cdots !}_{n+1} c, \underbrace{! ! \cdots !}_{n} c: \ldots: ! c: c: X)$ for any $c: X \in \mathrm{CS}$ with $\underbrace{! ! \cdots !}_{n+1} c \in \operatorname{Sub}(t)$

If the logic contains the $\mathrm{j} 4$ axiom, we add

- $(! s, s: X)$ for any $(s, X) \in \mathcal{E}_{t}^{n}(w)$ with $! s \in \operatorname{Sub}(t)$

- $(s, X)$ for any $(s, X) \in \mathcal{E}_{t}^{n}(v)$ with $R(v, w)$ and $s \in \operatorname{Sub}(t)$

All the sets $\mathcal{E}_{t}^{i}(w)$ are finite. As $W$ and $\operatorname{Sub}(t)$ are finite, there is an $n$ easily computable from the size of $W$ and the length of $t$ such that $\mathcal{E}_{t}^{n}(w)=\mathcal{E}_{t}^{i}(w)$ for all $i \geq n$. Furthermore, we have $\mathcal{E}(t, B, w)$ if and only if $B$ unifies with some $X$ such that $(t, X) \in \mathcal{E}_{t}^{n}(w)$. Thus, the relation $\mathcal{E}(t, B, w)$ is decidable.

\section{Corollary 22 (Decidability).}

1. Any justification logic in $\left\{\mathrm{J}_{\mathrm{CS}}, \mathrm{JT}_{\mathrm{CS}}, \mathrm{J}_{\mathrm{CS}}, \mathrm{LP}_{\mathrm{CS}}\right\}$ with a decidable schematic CS is decidable.

2. Any justification logic in $\left\{\mathrm{JD}_{\mathrm{CS}}, \mathrm{JD}_{\mathrm{CS}}\right\}$ with a decidable, schematic and axiomatically appropriate $\mathrm{CS}$ is decidable.

Proof. All logics presented are obviously recursively enumerable. By Corollary 18, Lemma 20 and Lemma 21 all logics presented satisfy the conditions of Theorem 19 and are, therefore, decidable. 


\section{The Case of Common Knowledge}

While the finiteness of the sets of worlds is a key feature of filtrations, the finite bases of our examples are due to the specific setup of the models and are by no means a necessary property of filtrations. On the other hand, if we start with a logic $L_{C S}$ which we already know to be sound and complete with respect to a class of finite models, we can adapt the construction we used to finitely base the evidence function for the filtrations.

Definition 23. Let $\mathcal{M}=(W, R, \mathcal{E}, \nu)$ be a model and $\Phi$ some set of formulae that is closed under subformulae. The $\Phi$-generated submodel $\mathcal{M} \uparrow \Phi$ of $\mathcal{M}$ is defined as $(W, R, \mathcal{E}\lceil\Phi, \nu \uparrow \Phi)$ where

1. $\mathcal{E} \uparrow \Phi$ is the minimal evidence relation based on $\mathcal{B}_{\Phi}$ where

$$
\mathcal{B}_{\Phi}(t, B, w) \text { if and only if } t: B \in \Phi \text { and } \mathcal{E}(t, B, w)
$$

2. $\nu \uparrow \Phi$ is given by

$$
(\nu \mid \Phi)(p)= \begin{cases}\{w \mid w \in \nu(p)\} & \text { if } p \in \Phi \\ \emptyset & \text { otherwise }\end{cases}
$$

Like in the case for filtrations we get the following lemma.

Lemma 24. Let $\mathcal{M}=(W, R, \mathcal{E}, \nu)$ be a model, $\Phi$ a set of formulae closed under subformulae, and $\mathcal{M} \uparrow \Phi$ the $\Phi$-generated submodel of $\mathcal{M}$. Then for all worlds $w$ in $\mathcal{M}$ and formulae $A \in \Phi$ we have

$$
\mathcal{M} \uparrow \Phi, w \Vdash A \text { if and only if } \mathcal{M}, w \Vdash A .
$$

Proof. The proof is by induction on $A$. The case for atomic propositions is immediate by the definition of $\nu \uparrow \Phi$ and the cases for boolean connectives follow immediately by induction hypothesis. Let us consider the case when $A$ is $t: B$.

So assume $\mathcal{M} \uparrow \Phi, w \Vdash t: B$. We get $(t, B, w) \in \mathcal{E} \uparrow \Phi$ and $\mathcal{M} \uparrow \Phi, v \Vdash B$ for all $v \in W$ with $R(w, v)$. The latter gives us $\mathcal{M}, v \Vdash B$ by induction hypothesis whereas from the former we get $(t, B, w) \in \mathcal{E}$ as both $\mathcal{E}$ and $\mathcal{E}\lceil\Phi$ are based on $\mathcal{B}_{\Phi}$ and $\mathcal{E} \uparrow \Phi$ is minimal with that property and hence $\mathcal{E} \uparrow \Phi \subseteq \mathcal{E}$. So we have $\mathcal{M}, w \Vdash t: B$.

For the other direction assume $\mathcal{M}, w \Vdash t: B$. We have thus $\mathcal{E}(t, B, w)$ and $\mathcal{M}, v \Vdash B$ for all $v \in W$ with $R(w, v)$. Again, the latter gives us $\mathcal{M} \uparrow \Phi, v \Vdash B$ by induction hypothesis and by the definition of $\mathcal{E} \uparrow \Phi$ we immediately get $(t, B, w) \in \mathcal{E}\lceil\Phi$ from the former and thus $\mathcal{M} \uparrow \Phi, w \Vdash t: B$.

We can use this technique (adapted to the multi-agent case) to establish decidability for the justification logic with common knowledge $\operatorname{LP}_{h}^{C}$ that was introduced in [9].

The logic $L P_{h}^{C}$ is a multi-agent version of LP ${ }_{C S}$ with additional axioms and operations on terms to deal with mutual and common knowledge. There are 
separate sets of terms for each agent $i \in\{1, \ldots, h\}$ as well as for mutual knowledge $\mathrm{E}$ and for common knowledge $\mathrm{C}$. The different kinds of knowledge suggest a slight change of notation. We will write $[t]_{\circledast} B$ to mean " $t$ is a justification term of type $\circledast$ for $B$ ", where $\circledast \in\{1, \ldots, h, \mathrm{E}, \mathrm{C}\}$. Furthermore $*$ will always denote an element of $\{1, \ldots, h, \mathrm{C}\}$.

The logic $\mathrm{LP}_{h}^{\mathrm{C}}(\mathrm{CS})$ is given by the following axioms as well as the rules for modus ponens and axiom necessitation (for a given constant specification CS):

1. finitely many schemes axiomatizing classical propositional logic

2. $[t]_{*}(A \rightarrow B) \rightarrow\left([s]_{*} A \rightarrow[t \cdot s]_{*} B\right)$

3. $[t]_{*} A \vee[s]_{*} A \rightarrow[t+s]_{*} A$

(application)

4. $[t]_{i} A \rightarrow A$

5. $[t]_{i} A \rightarrow[! t]_{i}[t]_{i} A$

6. $\left[t_{1}\right]_{1} A \wedge \cdots \wedge\left[t_{h}\right]_{h} A \rightarrow\left[\left\langle t_{1}, \ldots, t_{h}\right\rangle\right]_{\mathrm{E}} A$

7. $[t]_{\mathrm{E}} A \rightarrow\left[\pi_{i} t\right]_{i} A$

8. $[t]_{\mathrm{C}} A \rightarrow\left[\mathrm{cCl}_{1}(t)\right]_{\mathrm{E}} A, \quad[t]_{\mathrm{C}} A \rightarrow\left[\mathrm{cCl}_{2}(t)\right]_{\mathrm{E}}[t]_{\mathrm{C}} A$

9. $A \wedge[t]_{\mathrm{C}}\left(A \rightarrow[s]_{\mathrm{E}} A\right) \rightarrow[\operatorname{ind}(t, s)]_{\mathrm{C}} A$

(reflexivity) (inspection) (projection) (co-closure) (induction)

The semantics for $\operatorname{LP}_{h}^{C}$ is given by models

$$
\mathcal{M}=\left(W, R_{1}, \ldots, R_{h}, \mathcal{E}_{1}, \ldots, \mathcal{E}_{h}, \mathcal{E}_{\mathrm{E}}, \mathcal{E}_{\mathrm{C}}, \nu\right)
$$

where $R_{i}$ are reflexive, transitive accessibility relations on $W$ and $\mathcal{E}_{\circledast}$ are evidence relations satisfying closure conditions modeled on the axioms of LP ${ }_{h}^{C}$ analogous to the logics presented in Section $2 .{ }^{1}$ Furthermore we define $R_{\mathrm{E}}:=\bigcup_{i=1}^{h} R_{i}$ and $R_{\mathrm{C}}$ as the transitive closure of $R_{\mathrm{E}}$. A formula being satisfied at a given world is then defined as before with the following crucial case for the formula being of the form $[t]_{\circledast} B$

1) $\mathcal{E}_{\circledast}(t, B, w)$ holds and

2) $\mathcal{M}, v \Vdash B$ for all $v \in W$ with $(w, v) \in R_{\circledast}$.

Using a canonical model construction we can show the soundness and completeness of $\mathrm{LP}_{h}^{\mathrm{C}}$ with respect to this class of models and as an immediate corollary of this construction (see [9, Theorem 20]) we obtain

Theorem 25. $\mathrm{LP}_{h}^{\mathrm{C}}(\mathrm{CS})$ is sound and complete with respect to the class of singleton models for $\mathrm{LP}_{h}^{\mathrm{C}}(\mathrm{CS})$.

We can easily adapt the $\Phi$-generated submodels from Definition 23 and Lemma 24 to the multi-agent case and turn these singleton models into finitary models. Obviously the class of these finitary, singleton models is recursively enumerable and adapting Lemma 21 to the multi-agent case shows that LP $_{h}^{C}$ satisfies the conditions of Theorem 19. Decidability of $L P_{h}^{C}$ then follows as in the previous section.

Theorem 26. $\operatorname{LP}_{h}^{\mathrm{C}}(\mathrm{CS})$ with a decidable schematic CS is decidable.

\footnotetext{
${ }^{1}$ Note that these closure conditions are very similar and also give rise to a monotone operator as before. This is crucial in adapting the previous proofs to the multi-agent case.
} 


\section{Conclusion}

We have presented a uniform method of proving decidability for justification logics using a refinement of the finite model property. In order to achieve this property, we have adapted the modal techniques of filtration and generated submodels to justification logics. Apart from reproving the known decidability re-

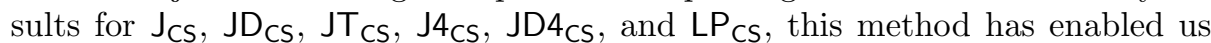
to establish the decidability of the justification logic with common knowledge introduced in [9].

The main difference from the modal case is the presence of an additional element in models called evidence relation. As evidence relations are in general infinite objects, the filtration has to be performed in such a way that apart from finitizing the set of worlds, also the evidence relation is finitely representable. This finite representation is achieved by using least fixed points of a certain monotone operator that can be read off the axioms of the logic. The existence of the least fixed point is guaranteed when the operator is monotone, which is the case for all the logics considered. Some logics, e.g. justification logics with negative introspection, however, give rise to non-monotone operators. Proving decidability for them requires more involved techniques, see [20].

\section{References}

1. Artemov, S.N.: Operational modal logic. Tech. Rep. MSI 95-29, Cornell University (Dec 1995)

2. Artemov, S.N.: Explicit provability and constructive semantics. Bulletin of Symbolic Logic 7(1), 1-36 (Mar 2001)

3. Artemov, S.N.: The logic of justification. The Review of Symbolic Logic 1(4), 477513 (Dec 2008)

4. Artemov, S.N., Fitting, M.: Justification logic. In: Zalta, E.N. (ed.) The Stanford Encyclopedia of Philosophy. Fall 2011 edn. (2011), http://plato.stanford.edu/ archives/fall2011/entries/logic-justification/

5. Artemov, S.N., Kuznets, R.: Logical omniscience as a computational complexity problem. In: Heifetz, A. (ed.) Theoretical Aspects of Rationality and Knowledge, Proceedings of the Twelfth Conference (TARK 2009). pp. 14-23. ACM, Stanford University, California (Jul 6-8, 2009)

6. Blackburn, P., Rijke, M., Venema, Y.: Modal logic. Cambridge tracts in theoretical computer science, Cambridge University Press (2002)

7. Brünnler, K., Goetschi, R., Kuznets, R.: A syntactic realization theorem for justification logics. In: Beklemishev, L.D., Goranko, V., Shehtman, V. (eds.) Advances in Modal Logic, Volume 8, pp. 39-58. College Publications (2010)

8. Bucheli, S., Kuznets, R., Renne, B., Sack, J., Studer, T.: Justified belief change. In: Arrazola, X., Ponte, M. (eds.) LogKCA-10, Proceedings of the Second ILCLI International Workshop on Logic and Philosophy of Knowledge, Communication and Action, pp. 135-155. University of the Basque Country Press (2010)

9. Bucheli, S., Kuznets, R., Studer, T.: Justifications for common knowledge. Journal of Applied Non-Classical Logics 21(1), 35-60 (Jan-Mar 2011) 
10. Bucheli, S., Kuznets, R., Studer, T.: Partial realization in dynamic justification logic. In: Beklemishev, L.D., de Queiroz, R. (eds.) Logic, Language, Information and Computation, 18th International Workshop, WoLLIC 2011, Philadelphia, PA, USA, May 18-20, 2011, Proceedings, Lecture Notes in Artificial Intelligence, vol. 6642, pp. 35-51. Springer (2011)

11. Fitting, M.: The logic of proofs, semantically. Annals of Pure and Applied Logic 132(1), 1-25 (Feb 2005)

12. Kuznets, R.: On the complexity of explicit modal logics. In: Clote, P.G., Schwichtenberg, H. (eds.) Computer Science Logic, 14th International Workshop, CSL 2000, Annual Conference of the EACSL, Fischbachau, Germany, August 21-26, 2000, Proceedings, Lecture Notes in Computer Science, vol. 1862, pp. 371-383. Springer (2000)

13. Kuznets, R.: Complexity Issues in Justification Logic. Ph.D. thesis, CUNY Graduate Center (May 2008)

14. Kuznets, R.: Self-referential justifications in epistemic logic. Theory of Computing Systems 46(4), 636-661 (May 2010)

15. Lemmon, E.J., Scott, D.S.: An Introduction to Modal Logic. B. Blackwell, Oxford (1977)

16. Mkrtychev, A.: Models for the logic of proofs. In: Adian, S., Nerode, A. (eds.) Logical Foundations of Computer Science, 4th International Symposium, LFCS'97, Yaroslavl, Russia, July 6-12, 1997, Proceedings, Lecture Notes in Computer Science, vol. 1234, pp. 266-275. Springer (1997)

17. Pacuit, E.: A note on some explicit modal logics. In: Proceedings of the 5th Panhellenic Logic Symposium. pp. 117-125. University of Athens, Athens, Greece (Jul 25-28, 2005)

18. Post, E.L.: Recursively enumerable sets of positive integers and their decision problems. Bull. Am. Math. Soc. 50, 284-316 (1944)

19. Segerberg, K.: An Essay in Classical Modal Logic. Uppsala,Filosofiska Föreningen Och Filosofiska Institutionen Vid Uppsala Universitet (1971)

20. Studer, T.: Decidability for some justification logics with negative introspection (2011), http://www.iam.unibe.ch/ tstuder/papers/J5_Decidability.pdf, preprint 